\title{
Les lectures littéraires : évolution et enjeux d'un concept
}

Jean-Louis Dufays

\section{(2) OpenEdition}

1 Journals

Édition électronique

URL : http://journals.openedition.org/trema/1579

DOI : 10.4000/trema.1579

ISSN : 2107-0997

Éditeur

Faculté d'Éducation de l'université de Montpellier

\section{Édition imprimée}

Date de publication : 1 octobre 2002

Pagination : 5-16

ISSN : 1167-315X

\section{Référence électronique}

Jean-Louis Dufays, « Les lectures littéraires : évolution et enjeux d'un concept », Tréma [En ligne],

19 | 2002, mis en ligne le 01 octobre 2002, consulté le 19 avril 2019. URL : http:// journals.openedition.org/trema/1579; DOI : 10.4000/trema.1579

Ce document a été généré automatiquement le 19 avril 2019

Trema 


\title{
Les lectures littéraires : évolution et enjeux d'un concept ${ }^{1}$
}

\author{
Jean-Louis Dufays
}

\section{Introduction}

1 Mon propos dans cette communication est d'approfondir le cadre épistémologique dans lequel prend place l'enseignement de la lecture, de la littérature et de la lecture littéraire. En partant de l'analyse d'une étiquette couramment utilisée dans ce domaine de l'enseignement de la langue première, je voudrais interroger les options et les enjeux que celles-ci véhiculent. Je suis donc guidé ici par un but de clarification et d'explicitation, mais aussi par le désir de jeter un pont entre la théorie littéraire et la didactique de la littérature, ainsi qu'entre cette dernière et la didactique du français dans son ensemble.

2 Mon point de départ réside dans la lecture de plusieurs publications récentes qui utilisent, tantôt pour la préconiser, tantôt pour la proscrire, la notion de «lecture littéraire »;

- l'enquête de Baudelot, Cartier et Detrez Et pourtant ils lisent (1999) ;

- l'article de Jean-Louis Dumortier « La lecture littéraire : le pour et le contre » (2001a) et son livre Lire le récit de fiction (2001b) ;

- le livre de Bertrand Daunay Éloge de la paraphrase (2002) ${ }^{2}$;

- la nouvelle édition du « Que sais-je ? » consacré à La didactique du français, publié par JeanMaurice Rosier (2002);

- et enfin deux ouvrages récemment publiés sous la direction de Catherine Tauveron, Comprendre et interpréter le littéraire à l'école et au-delà (2001) et Lire la littérature à l'école (2002).

3 Tous ces auteurs ou presque parlent de la lecture littéraire comme s'il s'agissait d'une pratique clairement définie et dont la nature ne posait pas de problème : ils en parlent au singulier, la définissent de manière unique («la lecture littéraire, c'est...»), non conflictuelle, sans évoquer l'existence de définitions ou de perceptions concurrentes. 

lecture littéraire savante (qu'on pratique au lycée et à l'université), qui a sa méthode et ses codes institutionnalisés", mais c'est pour aussitôt emprunter à Bertrand Gervais $(1993,1998)$ la définition de la lecture littéraire comme « lecture qui fait de la densité du sens son territoire de prédilection »:

"Il s'agit d'une lecture attentive au fonctionnement du texte et à sa dimension esthétique, d'une lecture soucieuse de débusquer des effets de sens non immédiats et de les faire proliférer, de débusquer des effets de non-sens pour leur trouver du sens, toutes opérations qui supposent la mobilisation d'une culture antérieurement construite et la création d'une culture nouvelle ( (Tauveron, 2002, p. 18).

7 C'est à cette même idée d'une lecture ancrée dans la densité du sens que se réfère Bertrand Daunay (2002) pour fonder sa critique de la « lecture littéraire» :

"La notion de slecture littéraire), qui donne de nouveaux motifs à des interdits qui ne l'avaient pas attendue, connaît une réelle fortune aujourd'hui. Cette notion n'est pas étrangère aux anciennes catégorisations. [...] elle permet de faire l'économie de la notion de littérarité - reconnue caduque si l'on s'en tient à des critères internes aux textes - mais elle en récupère le bénéfice, puisque, sous couvert d'une ouverture virtuelle à toutes sortes de textes, elle permet, dans les faits, de refonder une hiérarchisation implicite des textes, selon qu'ils offrent ou non matière à une lecture littéraire " (Daunay, 2002, p. 45).

Les positions de ces différents auteurs sont claires et apparemment convergentes. Pourtant, lorsqu'on lit de près ce qu'ils avancent et les travaux auxquels ils font référence, on s'aperçoit que: 1) la lecture littéraire ne fait nullement l'objet d'une représentation commune ; 2) il n'y a pas non plus d'accord sur la nécessité, voire sur la pertinence de cette notion dans l'enseignement - apprentissage.

Cela pose un problème pour les lecteurs de ces textes, en particulier pour les étudiants et les jeunes enseignants en quête de cadres épistémologiques cohérents, car, dans les ouvrages que je viens d'évoquer, les différentes conceptions de la lecture littéraire sont soit ignorées, soit gommées dans leurs spécificités. Ainsi, Rosier assimile Picard et Marghescou dont, comme on le verra, les modèles diffèrent assez fortement, et Dumortier classe dans une même catégorie les travaux de Descotes (1995), de Biard et Denis (1993), de Duquesne (1993) et ceux que j'ai publiés avec L. Gemenne et D. Ledur (1996) ${ }^{4}$, en omettant de préciser que les deux premiers ne traitent pas de la lecture littéraire mais seulement de la « lecture méthodique » et de la " didactique du texte littéraire ».

10 Il y a là en tout cas un double dissensus qu'il convient de creuser. Il scandera les deux parties de mon bref développement : j'étudierai tour à tour les définitions diverses qui 
sont données aujourd'hui de la lecture littéraire ${ }^{5}$ et les objections dont cette pratique ou du moins certaines de ses manifestations - fait l'objet.

\section{Quatre conceptions de la lecture littéraire}

\subsection{La lecture littéraire comme lecture des textes littéraires}

11 La première conception arrime la lecture littéraire à la prise en considération préalable du texte littéraire et de sa littérarité supposée. Cette conception continue donc de privilégier l'objet (la littérature ou le texte) par rapport à la pratique (la lecture) sans impliquer la reconnaissance d'un travail propre au lecteur: le texte prévaut indépendamment de sa réception, il reste un monument déjà saturé de sens et de valeur qu'il s'agit de révéler par une lecture qui s'applique à en célébrer les virtualités.

C'est là la conception la plus simple, la moins construite de la notion de "lecture littéraire », et elle n'entraîne aucun déplacement par rapport aux objets traditionnels de la discipline "français » et par rapport à l'activité du lecteur: la littérature (souvent conçue comme un corpus plus ou moins stable d'œuvres légitimées) continue d'exister d'un côté, et la lecture (souvent assimilée à un commentaire plus ou moins savant) de l'autre. Bien qu'elle ne fasse l'objet d'aucune modélisation théorique, c'est sans doute cette acception de la "lecture littéraire» qui est la plus répandue tant chez les enseignants que chez les chercheurs ${ }^{6}$. Son absence de spécificité permet cependant de se demander si l'emploi de l'étiquette « lecture littéraire » se justifie en l'occurrence.

En revanche, le fait de différencier un travail visant à l'appropriation et à la problématisation du fait littéraire et un travail sur la lecture comme activité investie de certaines qualités parait constitutif de l'équilibre de la discipline « français » (cf. : Legros, 1996, 1998 ; Dufays, 1999). Il existe en effet une complémentarité entre la construction de l'objet littérature et la pratique de cet objet, ou, si l'on préfère, entre la connaissance problématisée de l'objet et son exploitation dans des activités de réception.

\subsection{La lecture littéraire comme distanciation}

14 À l'idée d'une lecture littéraire centrée sur les propriétés du texte s'oppose celle d'une activité visant à investir des valeurs littéraires dans la lecture elle-même. Cette conception qui privilégie donc la pratique plutôt que l'objet est souvent solidaire d'une conception "intégrationniste » de la lecture et des textes dans la mesure où elle ne concerne pas nécessairement le seul corpus des textes littéraires ( $c f$. : Noël-Gaudeault, 1997, p. 11). Elle comporte elle-même trois modélisations distinctes selon qu'elle privilégie plutôt ce que j'appelle la distanciation, la participation psychoaffective ou le va-et-vient dialectique (Dufays, 1994, pp. 179-202).

La conception qui se fonde sur la distanciation peut être comparée à la lecture herméneutique dont parle Riffaterre (1982), à la lecture pseudo-référentielle de Stierle (1979) ou encore à la coopération interprétative d'Eco (1985). Elle trouve sa première formulation dans l'ouvrage de Marghescou (1974) Le concept de littérarité, qui n'emploie pas comme tel le terme de "lecture littéraire » mais décrit la manière dont la lecture littérariserait les textes en recourant à une triple opération: suspension de la valeur anecdotique (ou référentielle) du texte, manifestation de ses valeurs archétypales (ou symboliques), activation maximale de sa polysémie. Le premier à avoir donné à cette 
manière de lire le nom de «lecture littéraire » est Bertrand Gervais (1993 et 1998), qui définit cette pratique comme le passage d'une « régie de la progression » à une « régie de la compréhension », caractérisée par une exploration de toutes les virtualités du textes. Solidaires de l'esthétique « moderne », les valeurs privilégiées ici sont à la fois celles de la polysémie, de la subversion, de la fictionalité affichée, de la transgression et de la poéticité (Dufays, 1999, p. 91).

C'est à cette conception que se rattachent majoritairement les travaux - remarquables au demeurant - de didacticiens comme Catherine Tauveron $(1999,2001,2002)$ ou Annie Rouxel $(1996)^{7}$. Parce que cette manière de lire va de pair avec l'accès à la symbolisation, la mobilisation d'activités cognitives et culturelles variées, la construction d'un sens et d'une culture communs, ses enjeux didactiques sont sans conteste fondamentaux, et l'on s'avise aujourd'hui de plus en plus qu'il convient de les développer dès le plus jeune âge ${ }^{8}$. Il me semble cependant que, dans la mesure où ils se fondent sur l'arrachement aux pratiques spontanées et le contrôle des émotions subjectives, ils ne suffisent pas à définir une «lecture littéraire». D'une part, en effet, dans l'usage ordinaire, l'adjectif « littéraire » véhicule aussi l'idée d'une satisfaction d'ordre psychoaffectif; est-il possible et opportun de l'épurer de cette connotation? D'autre part, quand elle se fait exclusive, la posture de distanciation n'échappe pas aux accusations d'artifice scolastique et d'élitisme socioculturel ${ }^{9}$ et devient, pour beaucoup (Reuter, 1996b; Baudelot, Cartier et Detrez, 1999 ; Dumortier, 2001 ; Daunay, 2002), la source d'un écart dommageable entre la lecture scolaire et les pratiques sociales de référence.

\subsection{La lecture littéraire comme participation}

On ne s'étonnera pas, dès lors, si, à l'inverse de la conception qui vient d'être évoquée, de nombreux auteurs affirment que la posture qu'il conviendrait de valoriser en priorité à l'égard de la littérature à l'école est la lecture la plus « ordinaire ", celle qui privilégie l' «illusion référentielle ", l'implication psychoaffective du lecteur dans les référents du texte et qui correspond en gros à la lecture heuristique de Riffaterre, à la lecture quasipragmatique de Stierle, ou encore à ce qu'Eco appelle l'utilisation du texte et Gervais la régie de la progression. Cette conception rejoint à la fois celle de la lecture comme braconnage, comme "art de faire ", d'« inventer le quotidien ", dont parlait Michel de Certeau (1980), le « droit au bovarysme » dont parle Daniel Pennac (1992) et l'éloge de la paraphrase cher à Bertrand Daunay (2002), mais, à ma connaissance, le seul auteur à l'avoir explicitement assimilée à la « lecture littéraire » est Christian Poslaniec dans son essai de 1992 De la lecture à la littérature ${ }^{10}$.

es valeurs associées à cette conception de la lecture sont celles de la lisibilité (l'unité, la cohérence), de la conformité aux codes génériques, du rapport à la réalité, de la conformité éthique et de la référentialité (Dufays, 1999, p. 91). Ses enjeux didactiques sont la valorisation des réceptions spontanées (notamment des commentaires paraphrastiques), la mise en œuvre des ressources de l'émotion, de l'imagination, de la passion, de la subjectivité, mais aussi la relativisation de la littérature canonique et du sens commun, le travail sur des objets multiples, non propres à la littérature légitimée et la lecture individuelle vue comme lieu d'un « braconnage » intelligent.

Il n'est pas douteux que ces enjeux sont tout aussi essentiels que ceux de la lecture distanciée. Pour les lecteurs en difficulté, ils apparaissent même vitaux, dans la mesure où ils rejoignent et légitiment leur rapport au livre (Burgos, 1994), la lecture n'étant 
porteuse de sens à leurs yeux que lorsqu'elle est d'abord "pragmatiquement ancrée » (Lahire, 1993). Comme le souligne Dumortier, "Si tous ceux qui, dans le cadre de l'institution scolaire, sont chargés de la formation littéraire, plutôt que de dénigrer les objets et les manières de lire de ces lecteurs indifférents à la chose littéraire, s'intéressaient à ce que ces derniers ont acquis en renouvelant l'expérience du plaisir de lire leurs écrivains de prédilection, nous pensons qu'ils pourraient circonscrire ce terrain d'entente sans lequel il n'est pas de dialogue envisageable à propos de littérature, pas de sensibilisation possible à la question de la valeur artistique» (2001b, p. 181).

20 L'assimilation exclusive de la lecture littéraire à cette manière de lire ${ }^{11}$ souffre cependant de deux handicaps : 1) cette pratique est beaucoup plus souvent qualifiée d'ordinaire que de littéraire : il parait donc difficile de lui réserver l'emploi de l'étiquette «littéraire »; 2) ce mode de lecture, qui privilégie surtout l'éveil du goût de lire et l'entretien des appétences, n'est pas en soi porteur d'apprentissages, du développement de compétences nouvelles.

Dès lors, moins encore que la " distanciation ", la " participation » ne parait en mesure de revendiquer à elle seule l'appellation « lecture littéraire ». J'en viens ainsi à la quatrième conception, qui, comme on l'aura compris, s'efforce, quant à elle, d'intégrer les deux précédentes sans rien leur ôter de leur essence et de leur exigence.

\subsection{La lecture littéraire comme va-et-vient dialectique}

L'origine de cette dernière définition se trouve chez Picard qui la développa en 1986 dans La lecture comme jeu. Son approche peut être résumée en deux points : 1) tout lecteur est triple : liseur (instance physique, sensorielle), lu (instance psychoaffective, émotionnelle) et lectant (instance intellectuelle, rationnelle, interprétative); 2) la lecture se fait littéraire lorsqu'elle met en tension des valeurs opposées qui appartiennent aux sphères respectives du lu et du lectant : sens vs significations, conformité vs subversion, réalité vs fiction (à quoi l'on pourrait ajouter bienséance vs transgression, fonction référentielle vs fonction poétique, etc.).

Certes, comme Daunay (2002, p. 47-53) l'a bien noté, Picard se contredit quand il prétend illustrer ses principes dans des analyses de textes ou de pratiques particulières : à ce moment, seul le lectant est valorisé, et ce qui relève du lu et du liseur devient marginal, voire objet de mépris. Rien n'oblige toutefois de tenir les travaux de Picard comme la référence unique en la matière, et rien n'empêche de s'inspirer librement de son modèle théorique pour développer une conception cohérente de la didactique de la littérature. En l'occurrence, je me permets de rappeler ici la modélisation de la lecture littéraire que j'ai moi-même proposée dans mon livre Stéréotype et lecture (1994) puis dans l'ouvrage que j'ai publié avec L. Gemenne et D. Ledur (1996a), qui proposait de fonder sur cette base un programme global d'initiation à la « lecture littéraire ». Cette conception a inspiré les dernières instructions officielles de l'enseignement catholique en Belgique francophone, qui, à leur tour, préconisent la mise en œuvre d'une lecture littéraire conçue comme "une activité complexe de résolution de problèmes \& qui tresse incessamment des démarches complémentaires) »(Fésec, 2000, p. 11).

Les enjeux didactiques de cette conception de la lecture me semblent être de deux ordres. En premier lieu, elle oblige à penser ensemble, de manière systémique, le rapport entre l'ancrage et le désancrage du sens, la fonction référentielle et la fonction poétique, les rapports passionnel et rationnel, la subjectivité et l'intersubjectivité, la centration sur un 
corpus restreint et l'accueil d'un corpus ouvert, le privilège accordé aux valeurs "modernes" et celui accordé aux valeurs "classiques", qui sont perçus comme deux pôles au sein d'un continuum et deux composantes à l'œuvre dans toute lecture. Ce faisant, cette conception évite la rupture avec la «lecture ordinaire», puisqu'elle se définit comme une accentuation de tensions qui la caractériseraient en permanence : elle intègre la « lecture savante » et la « lecture ordinaire » dans une même activité sans pour autant ignorer les tensions qui s'y jouent entre deux polarités.

RINCÉ D., LECHERBONNIER B. et MITTERAND H. (dir.) : Littérature. Textes et documents XIXe. Paris, Nathan, 1986, p. 515, 592 pp.

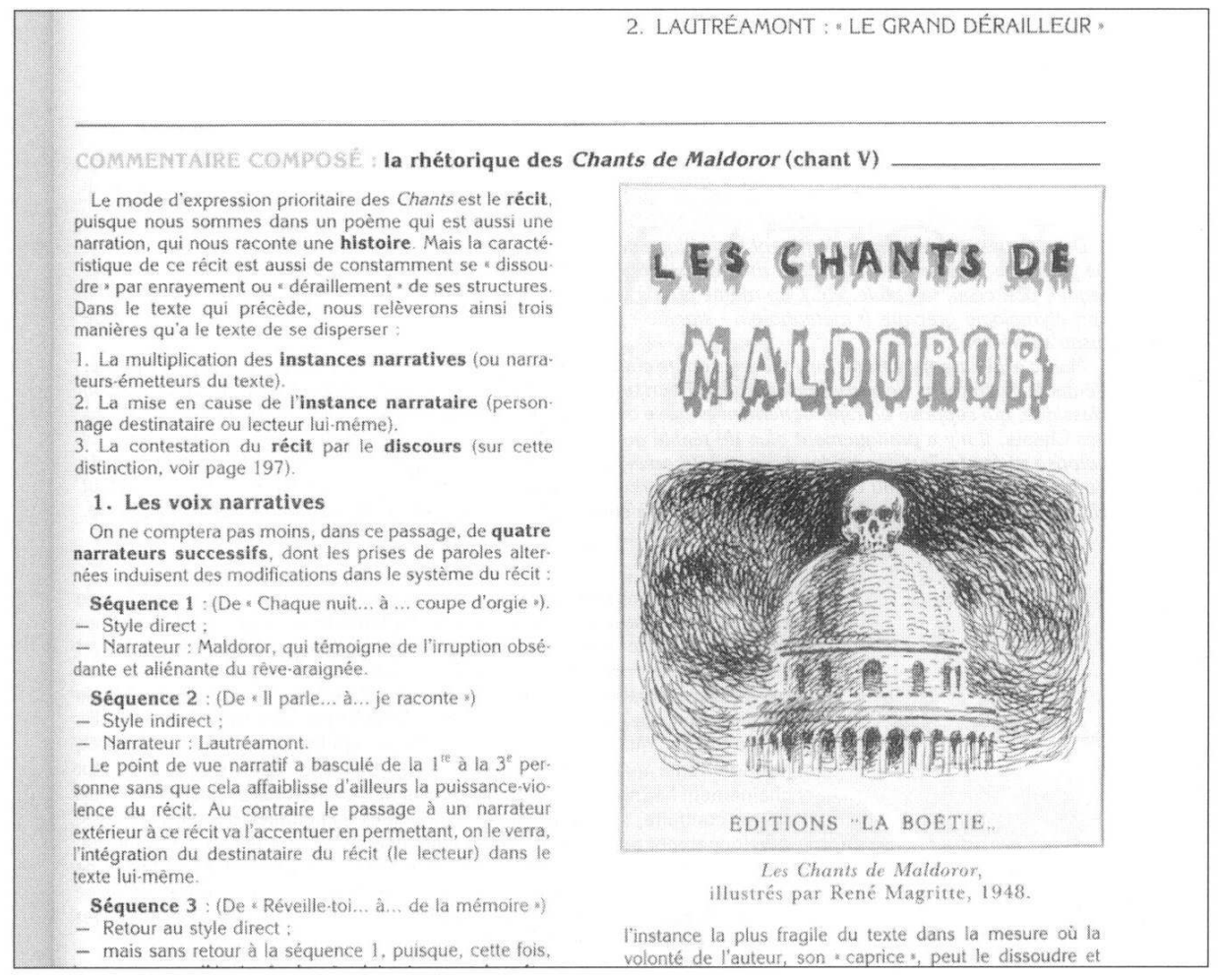

En second lieu, cette conception peut être reliée aux trois temps de l'apprentissage tels qu'ils ont été formalisés (par Meirieu, puis par bien d'autres) dans le dispositif de la " séquence didactique » : au va-et-vient entre l'ancrage, le désancrage et le réancrage de la lecture dans l'univers référentiel correspondent en effet l'alternance entre les moments de contextualisation (par une production initiale), de décontextualisation (via des ateliers de structuration) et de recontextualisation (par une production finale) des apprentissages.

Pour emprunter un terme proposé ici même par G. Mathis ${ }^{12}$, cette conception constitue dès lors le lieu possible d'une "reliance", certes conflictuelle, mais opératoire entre différents éléments et valeurs de la discipline "français». On voit bien aussi ce qui différencie cette approche dialectique de celles qui, privilégiant un seul des deux pôles, font de la lecture littéraire le lieu d'une hiérarchisation : les deux perspectives s'originent dans des théories fondatrices différentes (Marghescou et Gervais vs Picard et Dufays) ${ }^{13}$ qui conçoivent d'une tout autre manière les relations entre les lectures "littéraire » et « ordinaire ». 


\section{Deux objections contre la lecture littéraire} isante, lettrée, inaccessible à la plupart des élèves (Reuter, 1996b · Baudelot, Cart et Detrez, 1999 ; Dumortier, 2001a et b ; Daunay, 2002), ou, à l'inverse, une pratique axée sur la subjectivité, sur l'affectivité, mais dont le caractère socioculturel et construit est généralement occulté (Privat, 1993).

31 À l'évidence, cette objection s'adresse à ce que j'ai appelé respectivement la distanciation et la participation, mais elle ne concerne pas le va-et-vient dialectique. Il est d'ailleurs frappant de constater que c'est à cette dernière conception que se rattachent ceux qui se posent en adversaires de la « lecture littéraire » : ce qu'ils rejettent, c'est l'ambiguïté liée aux emplois multiples de la notion et aux effets pervers qui risquent d'en résulter, mais à travers leur méfiance se pointe clairement l'appel à une représentation dialectique de l'acte de lire.

32 C'est bien, par exemple, de dialectique entre le lu et le lectant, entre la participation et la distanciation, qu'il est question chez l' « amateur éclairé » que Dumortier appelle de ses vœux (2001b, p. 180-188), et Reuter ne disait pas autre chose dans un article où il insistait cependant sur la difficulté de l'entreprise :

"Pour réussir cela (la canonisation de textes et de principes de lecture), il faut d'une façon ou d'une autre réduire la tension entre lecture courante et lecture esthétisante ou du moins la transformer pour qu'elle n'apparaisse pas en tant que telle. [...] Cependant, cet effort 
d'enseignement ne peut être que perpétuellement difficultueux et conflictuel dans la mesure où ce travail scolaire est constamment remis en cause aussi bien par les résistances de certains élèves qui importent leur mode de lecture que par les théories de la lecture ou par la logique même du champ littéraire dans sa recherche d'autonomie " (Reuter, 1996b, p. 39).

On peut toutefois craindre qu'en s'opposant à toute forme de didactisation du concept de lecture littéraire, ces auteurs ne se fassent, malgré eux, les complices d'une représentation de la lecture tout aussi réductrice que celle qu'ils entendent rejeter : de la dénonciation de la lecture esthétisante à la valorisation exclusive de la paraphrase, de la lecture «fonctionnelle» ou encore de la «lecture ordinaire» ou "courante», il n'y a qu'un pas que les enseignants sensibles aux enjeux stratégiques de l'adjectif « littéraire » auront tôt fait de franchir. À tout prendre, entre un modèle qui précise et dynamise la fonction du littéraire au sein d'une conception complexe de la lecture et un autre qui préconise la même attitude sans assumer sa nature littéraire, n'est-ce pas le second qui offre le plus de prise à des malentendus et à des dérives dommageables?

Mais cette seconde objection a du moins le mérite de faire apparaître les conditions nécessaires pour que la notion de «lecture littéraire » puisse faire l'objet d'un emploi pertinent dans le champ didactique, conditions qui me semblent être de deux ordres :

1. Sa définition se doit d'être clarifiée et de se faire clairement intégrative, dialectique par rapport aux opérations de la lecture dite « ordinaire ». Autrement dit, il s'agit de prendre au sérieux l'idée forte de Picard selon laquelle toute lecture est plurielle, et de penser la relation entre lecture "ordinaire » et «lecture littéraire » sur le mode du continuum plutôt que de la rupture: on admettra alors que toute lecture s'avère plus ou moins « ordinaire » et plus ou moins "littéraire » selon l'intensité avec laquelle elle met en tension des postures axiologiques opposées.

2. Cette définition se doit d'aller de pair avec une pratique d'enseignement elle-même dialectique, axée sur différentes activités complémentaires, les unes relevant de la participation, les autres privilégiant la distanciation (réflexion sur le fait littéraire, transmission de connaissances littéraires utiles, développement de compétences interprétatives) ${ }^{14}$.

En somme, la double question posée par cette brève réflexion peut être résumée comme suit : la didactique du français a-t-elle besoin ou non du concept de « lecture littéraire », et si, comme je le pense, c'est le cas, a-t-elle intérêt à définir cette lecture comme une pratique opposée à la « lecture ordinaire » ou au contraire comme une activité qui intègre et accentue une tension inhérente à toutes les lectures? À travers la réponse, c'est tout le regard de l'enseignant non seulement sur la lecture et la littérature, mais aussi sur l'élève et ses apprentissages qui est en jeu.

\section{BIBLIOGRAPHIE}

BAUDELOT C. et CARTIER M. : « Lire au collège et au lycée : de la foi du charbonnier à une pratique sans croyance ", in Actes de la recherche en sciences sociales.1998, p. 123. 
BAUDELOT C, CARTIER M. et DETREZ C. : Et pourtant ils lisent.... Paris, Seuil, 1999.

BIARD J. et DENIS F. : Didactique du texte littéraire.Paris, Nathan (Perspectives didactiques), 1993.

BURGOS M. : « La lecture des adolescents : identification et interprétation », in L'École des Lettres $1^{\text {er }}$ cycle. $\mathrm{N}^{\circ} 12-13,1994$, pp. 37-40.

DAUNAY B. : « La lecture littéraire : les risques d'une mystification », in Recherches.1999, N

- 30, pp. 29-59.

Éloge de la paraphrase, Vincennes, Presses universitaires de Vincennes.

DE CERTEAU M. : L'invention du quotidien. I. Arts de faire. Paris, Gallimard (Folio Essais, N

○ 146) (éd. orig. : 1980), 1990.

DESCOTES M. et al. : Lire méthodiquement des textes. Paris, Bertrand-Lacoste, 1995.

DUFAYS J.-L. : Stéréotype et lecture. Essai sur la réception littéraire. Liège, Mardaga (Philosophie et langage), 1994.

«Culture/compétence/plaisirs : la nécessaire alchimie de la lecture littéraire », in DUFAYS J.-L., GEMENNE L. et LEDUR D. 1996b, pp. 167-175.

« Les nouvelles approches didactiques facilitent-elles l'accès des élèves à la littérature ? ", in $\mathbf{L} \boldsymbol{e}$ français aujourd'hui - hors série, Lecteurs, littératures, enseignement.Actes du XI ${ }^{\mathrm{e}}$ congrès de l'AFEF, 1999, pp. 89-102.

«Les compétences littéraires en français langue maternelle ou première : état des lieux et essai de modélisation », in COLLÈS L., DUFAYS J.-L., FABRY G. et MAEDER C. (DIR.) : Didactique des langues romanes. Le développement de compétences chez l'apprenant, Actes du colloque de Louvain-la-Neuve, janvier 2000. Bruxelles, De Bœck (Savoirs en pratique), 2001, pp. 245-251.

DUFAYS J.-L., GEMENNE L. et LEDUR D. : Pour une lecture littéraire 1. Approches historique et théorique, propositions pour la classe de français. Bruxelles, De Bœck - Duculot (Formation continuée), 1996a.

DUFAYS J.-L., GEMENNE L. et LEDUR D. : Pour une lecture littéraire 2. Bilan et confrontations, Actes du colloque de Louvain-la-Neuve. Bruxelles, De Bœck - Duculot (Formation continuée), 1996b.

DUMORTIER J.-L. : « La lecture littéraire : le pour et le contre », in Éducation Formation. 2001a, 262.

DUMORTIER J.-L. : Lire le récit de fiction. Pour étayer un apprentissage : théorie et pratique. Bruxelles, De Boeck (Savoirs en pratique), 2001b.

DUQUESNE D. : Lecture méthodique, lecture littéraire. Théorie. Pratiques. Perspectives.Angers, CRDP des pays de Loire - CRDP de Maine et Loire, 1993.

ECO U. : Lector in fabula. La coopération interprétative dans les textes narratifs. Paris, Grasset (figures), 1985.

FESEC (Fédération de l'enseignement secondaire catholique) : Programme. Français. $3^{e}$ degré Humanités générales et technologiques.Bruxelles, FESEC, 2000.

GERVAIS B. : À l'écoute de la lecture.Montréal (Canada), VLB éditeur, 1993.

GERVAIS B. : Lecture littéraire et explorations en littérature américaine. Montréal (Canada), XYZ éditeur, 1998.

JOUVE V. : La lecture.Paris, Hachette (Contours littéraires). 1993. 
LAHIRE B. : La raison des plus faibles. Rapport au travail, écritures domestiques et lectures en milieux populaires. Lille, Presses universitaires de Lille, 1993.

LEGROS G. : « Au-delà des œuvres, la littérature ? », in Français 2000.№ 149-150. 1996, pp. $15-21$

LEGROS G. : « Enseigner aujourd'hui la littérature », in Le français aujourd'hui. $N^{\circ} 121$, 1998, pp. 12-17.

MARCOIN F. : « Former des lecteurs ou former des lettre ? ", in Le français aujourd'hui. $\mathrm{N}^{\circ}$ 121,1998, pp. 18-27.

MARGHESCOU M. : Le concept de littérarité. Essai sur les possibilités théoriques d'une science de la littérature. La Haye - Paris, Mouton, 1974.

NOËL-GAUDREAULT M. (dir.) : Didactique de la littérature. Bilan et perspectives.Montréal (Canada), Nuit Blanche, 1997.

PENNAC D. : Comme un roman.Paris, Gallimard, 1992.

PICARD M. : « La lecture comme jeu », in Poétique. N 58, 1984, pp. 253-273.

PICARD M. : La lecture comme jeu. Paris, Minuit, 1986.

POSLANIEC C. : Donner le goût de lire. Des animations pour faire découvrir aux jeunes le plaisir de la lecture. Paris, Sorbier, 1990.

POSLANIEC C. : De la lecture à la littérature.Paris, Sorbier, 1992.

PRIVAT J.-M. : «L'institution des lecteurs », in Pratiques.N 80, 1993.

REUTER Y : « Définir les biens littéraires? », inPratiques. №67, 1990.

REUTER Y. : "Éléments de réflexion sur la place et les fonctions de la littérature dans la didactique du français à l'école ", in Repères. $N^{\circ} 13,1996 \mathrm{~A}, \mathrm{pp} .7-25$.

REUTER Y. : « La lecture littéraire : éléments de définition », in DUFAYS J.-L., GEMENNE L. et LEDUR D. 1996b, pp. 33-41.

RIFFATERRE M. : «L'illusion référentielle », in BARTHES R., et al. :Littérature et réalité.Paris, Seuil (Points), 1982, pp. 91-118.

ROSIER J.-M. : La didactique du français.PARIS, P.U.F. (Que sais-je ?), 2002.

ROUXEL A. : Enseigner la lecture littéraire. Rennes, Presses universitaires de Rennes (Didact. français), 1996.

STIERLE K. : « Réception et fiction », in Poétique.N 39, 1979, pp. 299-320.

TAUVERON C. (dir.) : « Comprendre et interpréter le littéraire à l'école : du texte réticent au texte proliférant », in Repères. $N^{\circ}$ 19, 1999, pp. 9-38.

TAUVERON C. : Interpréter le littéraire à l'école et au-delà. Paris, INRP, 1999.

TAUVERON C. : Lire la littérature à l'école. Pourquoi et comment conduire cet apprentissage spécifique? De la GS au CM. Paris, Hatier, 1999. 


\section{NOTES}

1. Communication présentée à l'IUFM de Grenoble le 29 mars 2002. Il s'agit de la première version d'un texte à paraître sous une version revue et complétée dans les Actes des $3^{\mathrm{e}}$ rencontres des chercheurs en didactique de la littérature.

2. Ce livre intègre notamment le texte d'un article publié en 1999 dans la revue Recherches sous le titre "La lecture littéraire : les risques d'une mystification ".

3. Notons au passage que, dans le livre qu'ils ont publié l'année suivante (1999, pp. 157-167) avec

C. Detrez, Baudelot et Cartier emploient surtout le vocable de "lecture savante", dont les connotations leur paraissent manifestement plus claires.

4. Évoquant ces différents auteurs, Dumortier affirme: “quelles que soient les précautions qu'ils prennent pour ne pas tomber dans les travers de l'applicationnisme, leur démarche est, dans bien des cas, de type « descendant )" (2001b, p. 462).

5. Je n'ignore pas, en me livrant à cette tentative de classement, l'effet réducteur qui résulte inévitablement d'une telle catégorisation, et a fortiori de l'assimilation de tel ou tel auteur à telle ou telle conception. Je sais aussi que les positions de ceux dont je citerai ici le nom ont pu fluctuer, et s'avérer beaucoup plus complexes que ce que mon (trop bref) classement en laissera apparaître. J'accepte donc par avance d'intégrer toutes les nuances et les précisions de ceux qui s'estimeraient injustement traités, mais je demande en même temps à ces auteurs-là d'admettre qu'il existe bel et bien des différences non négligeables entre les différentes positions et que leur mise en lumière s'avérait indispensable pour une clarification de cette problématique et du champ disciplinaire qui en est à la fois le contexte et le fruit.

6. À preuve, la (très bonne au demeurant) revue, créée en 1996 par Vincent Jouve, qui a pour nom La lecture littéraire,ne paraît guère se revendiquer d'une définition particulière de la lecture.

7. Dans sa thèse de doctorat (inédite), Annie Rouxel insiste néanmoins sur l'idée que la lecture littéraire est un mouvement d'aller-retour entre des moments divers, parmi lesquels des moments de participation psychoaffective.

8. Cf.les nouveaux programmes français pour l'enseignement fondamental qui seront d'application a la rentrée 2002.

9. Rappelons, s'il le faut, que, pour nombre d'élèves (selon la récente enquête de l'OCDE, $28 \%$ des élèves de 15 ans en Belgique francophone), la lecture est perçue a priori comme une pratique hautement problématique : la première nécessité face à de tels publics est donc de leur donner accès au livre par des médiations socioculturelles (Privat, Reuter, Rosier) et psychoaffectives (Poslaniec, Pennac) adéquates.

10. Dans cet essai, Poslaniec fondait la définition de la lecture littéraire sur "l'étonnement" ou "l'admiration" que les textes suscitent chez leur lecteur, quel qu'il soit et quel que soit son régime de lecture (Poslaniec, 1992, pp. 90-91). Toutefois, cet auteur m'a dit avoir pris par la suite ses distances avec cette définition, jugée par lui-même trop peu distinctive.

11. Attitude qui, précisons-le, n'est pas le fait de Dumortier. Celui-ci, au contraire, insiste, comme moi, sur l'existence d'un continuum entre la démarche "pragmatiquement ancrée " et celle du lettre (2001, p. 180).

12. MATHIS G.: "Une discipline de reliance ", in J.-F. Massol (dir), Actes des $3^{e}$ rencontres des chercheurs en didactique de la littérature(références à compléter au moment de la publication).

13. Il est frappant au passage de constater que Gervais, qui a publié son premier livre Àl'écoute de la lecturesept ans après La lecture comme jeude Picard, ne s'est, jusqu'à présent, jamais situé par rapport à l'approche dialectique de ce dernier. En outre, jusqu'à son dernier livre, il n'avait 
manifestement pas lu mon essai Stéréotype et lecturequ'il prenait pour un ouvrage de didactique (cf. Gervais, 1998, p. 17, note 4).

14. J'ajoute que d'autres activités encore gagneraient à être axées sur le liseur de Picard en privilégiant les appropriations sensorielles (par l'écoute, la mise en voix, la manipulation de l'objet livre) des textes.

\section{AUTEUR}

\section{JEAN-LOUIS DUFAYS}

Université Catholique de Louvain, CEDILL 